\title{
Apolipoprotein and Lipid Composition of Plasma Lipoproteins in Neonates during the First Month of Life
}

\author{
J. P. VAN BIERVliet, M. ROSSENEU, J. BURY, H. CASTER, M. S. STUL, AND R. LAMOTE \\ Department of Pediatrics and of Clinical Biochemistry, A.Z. ST-JAN Ruddershove 10 B-8000 Brugge, Belgium
}

\begin{abstract}
In this study the lipid and apoprotein profiles were investigated in newborns at 0,7 , and 30 days of life. The plasma lipoproteins were separated both by ultracentrifugation and gel filtration in order to compare the patterns obtained by the two techniques. At birth, the apo $E$ concentration is comparable to that measured in adults, but its distribution among lipoproteins is significantly different as more than $\mathbf{8 0 \%}$ of the plasma apo $\mathrm{E}$ belongs to high-density lipoproteins (HDL). At 7 and 30 days the plasma apo $E$ concentrations are close to the values at birth, but a significant redistribution occurs from HDL to very low-density lipoproteins. By analogy with apo $B$, the plasma apo CIII concentration is low at birth and increases between 0 and 7 days by a factor of about two. Plasma triglycerides increase significantly during the first week of life so that the apo CIII increase is most pronounced in very low-density lipoproteins. These lipoproteins therefore become enriched in apo $\mathrm{E}$, apo CIII and triglycerides between 0 and 7 days. At birth, a distinct HDL fraction, enriched in apo E, apo AII and cholesterol (HDL E $_{\text {, }}$, could be detected. To compensate for the low LDL levels, this $\mathrm{HDL}_{\mathrm{E}}$ fraction might function as an additional source for cholesterol delivery to peripheral tissues via the apo $(B$, E) receptor. At later age, low-density lipoprotein synthesis is enhanced, apo $\mathrm{E}$ is transferred to very low-density lipoproteins, and cholesterol delivery via the $\mathrm{HDL}_{\mathrm{E}}$ becomes less important. These data demonstrate that significant differences occur in the plasma concentration and distribution of the apo CIII and $E$ proteins during the initial period of life, and that these apoproteins fulfill an important metabolic role. (Pediatr Res 20: 324-328, 1986)
\end{abstract}

\section{Abbreviations}

HDL, high-density lipoproteins

VLDL, very low-density lipoproteins

LDL, low-density lipoproteins

During the perinatal period, major shifts in nutritional supply cause changes in substrate utilization by the fetus and newborn. Unimpeded substrate flow from placenta to fetus is required to meet the energy requirements for growth and for fuel storage, primarily of glycogen and fat. During the intra- and postpartum periods, this constant flow is interrupted so that glycogen is required to maintain serum glucose levels. Glycogen stores are then depleted and active glycogenesis starts. In the perinatal

Received August 8, 1985; accepted December 4, 1985

Correspondence Dr. J. P. Van Biervliet, Department of Pediatrics and of Clinical Chemistry, A.Z. St-Jan Ruddershove 10, B-8000 Brugge, Belgium. period, fatty acid and ketone body oxidation become important energy sources, and during this period an adaptation to a high fat milk diet becomes therefore necessary.

These adaptations are controlled by changes in substrate flow and hormonal milieu of the fetus and newborn. Such changes $n$ substrate result in rapid shifts in the synthesis and metabolism of lipid transporting particles, whose composition then changes from that before birth.

In newborns, plasma lipid and lipoprotein patterns also are significantly different from those observed in adults, both in their concentration and distribution. Newborn plasma lipoproteins consist mostly of HDL, while VLDL and LDL are present at low concentrations only $(1-6)$. At birth, apoprotein concentrations are lower than in the adult, especially apo B and apo CIII $(1,3)$.

These patterns undergo a rapid evolution towards an adult profile between 0 and 30 days. Such changes take place at different rates and extent for the various lipids and apoproteins. In VLDL and LDL the lipid and apoprotein components increase most between 0 and 7 days, whereas in HDL such increases occur up to 30 days $(1,2)$. These variations also are reflected in the levels of the HDL subclasses (6).

In view of the compositional differences between newborn and adult lipoproteins, one can assume that specific pathways are involved in the metabolism of newborn lipoproteins. The relative importance and the metabolic function of specific lipoproteins, such as apo E-rich HDL, might vary significantly during the 1 st month of life. Innerarity et al. (7) have suggested that, by analogy with abetalipoproteinemic patients, apo E-rich HDL in neonates might deliver cholesterol to tissues via the apo $(\mathrm{B}, \mathrm{E})$ receptor.

In order to investigate in further detail the rapid and significant evolution of the lipoproteins in neonates, we studied the apoprotein composition of plasma lipoproteins in newborns at 0,7 , and 30 days. The relative proportion of the major (apo AI, AII, and B) and minor (apo CIII and E) apoproteins has been investigated after lipoprotein separation, both by ultracentrifugation and gel filtration. These data provide some insight into the synthetic and metabolic processes occurring at this early stage of life as well as information about the specific functions of the apoproteins within the various lipoprotein classes.

\section{SUBJECTS AND METHODS}

Subjects. Only healthy, full-term infants born after uneventful parturition, were included in the study. Cord-blood $(n=17)$ was obtained from the umbilical vein after clamping of the umbilical cord, immediately after birth. Samples at day $7(n=15)$ and day $30(n=10)$ were obtained from the same newborns by venapuncture after a 7-h fast. Blood was collected on sodium EDTA $1 \mathrm{~g} /$ liter and centrifuged immediately at $7^{\circ} \mathrm{C}$

Lipoprotein isolation. Lipoproteins were isolated by isopycnic ultracentrifugation from $0.3 \mathrm{ml}$ plasma (8). As apo $\mathrm{E}$ is released from lipoproteins during ultracentrifugation, $0.5 \mathrm{ml}$ was also 
fractionated by gel filtration on a Sepharose 6B-CL column, enabling the separation of triglyceride-rich lipoproteins, LDL, and HDL (9).

Apoprotein and lipid quantitation. Cholesterol, phospholipids, and triglycerides were assayed enzymically (8). Apo AI, apo AII, and apo $\mathrm{B}$ were quantitated by immunonephelometry $(1,2)$. Apoproteins CIII and $\mathrm{E}$ were assayed by a Sandwich-type enzyme linked immunosorbent assay (ELISA) $(9,10)$.

From these data the lipoprotein composition of each fraction was calculated. The sum of cholesterol, phospholipid, and triglycerides and the apo AI, AII, B, CIII, and E were taken as $100 \%$ for lipid and apoprotein respectively; apo CI, CII were not assayed.

All statistical analyses were carried out using either a paired or an unpaired Student's $t$ test.

\section{RESULTS}

Apoprotein CIII and E concentrations and distribution. At birth the plasma apo CIII concentration was approximately half the adult value (Table 1). It increased significantly between 0 and 7 days $(p<0.001)$ and remained subsequently constant. In contrast the apo E concentration at birth was comparable to the adult value. A significant increase was observed between 0 and 7 days $(p<0.001)$, followed by a significant decrease between 7 and 30 days $(p<0.01)$. At birth, $8 \pm 2 \%$ of plasma apo CIII and $81 \pm 9 \%$ of plasma apo $\mathrm{E}$ were present in $\mathrm{HDL}$ (Fig. 1). Between 0 and 7 days, apo CIII and apo $E$ increased in all lipoprotein classes, but most significantly in VLDL (Fig. 1, Table 1). Between 7 and 30 days the apo CIII distribution did not vary significantly, while the apo $\mathrm{E}$ concentration decreased in HDL, resulting in a further redistribution of apo $E$ toward the triglyceride-rich lipoproteins.

Lipid and apoprotein composition of VLDL and LDL. The lipid and apopotein profiles, obtained after plasma separation by gel chromatography, are shown in Figures 2 and 3. The concentrations of the VLDL apoprotein and lipid constituents increased between 0 and 30 days, although at a different rate for the various components (Figs. 4-6). The VLDL triglycerides increased 5fold between 0 and 7 days, and this increase persisted up to 30 days. The increase in phospholipid, cholesterol, and apoproteins was most pronounced between 0 and 7 days (Figs. 4-6).

In terms of percentages, the VLDL particles became enriched in triglycerides and depleted in phospholipids; they contained $48.5 \pm 5.6 \%$ triglycerides, $33.6 \pm 6.4 \%$ phospholipids, and 17.9 $\pm 9.3 \%$ cholesterol at birth, compared to $62.5 \pm 10.3 \%$ triglycerides, $22.2 \pm 6.4 \%$ phospholipids, and $15.3 \pm 5.1 \%$ cholesterol at 30 days. The percentage of total protein in VLDL (14.7 \pm $2.3 \%$ ) did not change significantly as a function of time, although its composition varied from $71.4 \pm 8.6 \%$ apo $\mathrm{B}, 21.7 \pm 5.1 \%$ apo CIII, and $6.6 \pm 2.2 \%$ apo $E$ at birth, to $65.1 \pm 5.9 \%$ apo $B$, $23.2 \pm 5.1 \%$ apo CIII, and $9.4 \pm 5.8 \%$ apo $E$ at 30 days.

In LDL, the lipid and the apoprotein concentrations increased significantly $(p<0.01)$ between 0 and 7 days (Figs. 4-6). Between
7 and 30 days, the major change consisted of a decrease of the triglyceride concentration, without any significant change in the LDL cholesterol and apo B concentrations.

Composition of the HDL subclasses. Gel filtration of plasma did not enable the subfractionation of $\mathrm{HDL}$ into $\mathrm{HDL}_{2}$ and $\mathrm{HDL}_{3}$ (Fig. 2). However, the immunological quantitation of apoproteins AI, AII, CIII, and E enabled the resolution of an apo E-rich HDL (defined as $\mathrm{HDL}_{\mathrm{E}}$ ) in the HDL peak (Fig. 3). As shown in Figures 5 and 6 the apoprotein composition of $H_{D L}$ changes as a function of newborn age. Apo AII and E are present in highest amounts at birth and subsequently decrease until 30 days. During the same period, the apo AI level increases while that of apo CIII remains constant. The high apo B and cholesterol content of this fraction are probably due to LDL contamination.

In the neonate, the apo AII/E molar ratio remains constant throughout the $\mathrm{HDL}_{\mathrm{E}}$ fraction, suggesting that it consists of discrete particles, whereas this ratio increases with decreasing particle size in the adult. The mean apo AII/E ratio increases from around 3.5 at birth up to 4.5 and 5.0 at 7 and 30 days, respectively. Within the major HDL fraction, a differential evolution of lipids and apoproteins was observed between 0 and 30 days. Between 0 and 7 days a significant increase in triglycerides was observed, while phospholipids and cholesterol increased most between 7 and 30 days. In terms of composition, HDL were enriched with triglycerides at 7 days, and their composition was close to that of adults at 30 days.

The evolution of the apoprotein distribution paralleled that of the HDL lipids; apo CIII and apo E increased most between 0 and 7 days, during which period apo AI and AII remained constant. Between 7 and 30 days, a pronounced increase of apo AI and apo AII was observed. The apoprotein composition of HDL was characterized by increased percentages of apo CIII and apo $\mathrm{E}$ at 7 days, while the composition at 30 days resembled that of adult lipoproteins. During this maturation process, the apo $\mathrm{AI} / \mathrm{AII}$ ratio in HDL increased significantly $(p<0.025)$ from a mean value of 1.2 at 0 and 7 days to 2.5 at 30 days. This value is comparable to that measured in eight adults. A plot of the apo

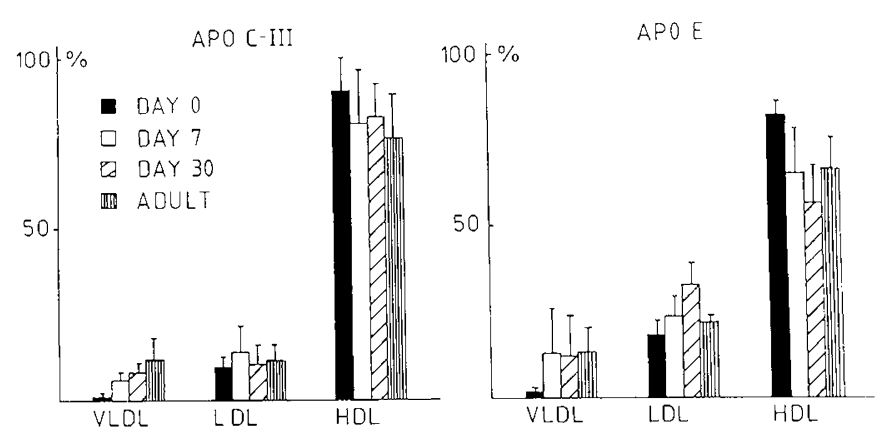

Fig. 1. Distribution of apo C-III and apo $\mathrm{E}$ in human plasma lipoproteins as a function of newborn age. Lipoproteins were separated by gel filtration on a Sepharose $6 \mathrm{~B}$ column.

Table 1. Apoprotein C-III and E total plasma concentrations $(\mathrm{mg} / \mathrm{dl}, \pm S E M)$ at birth $(n=17)$, day $7(n=15)$, and at day $30(n==$ 10) compared to adult values $(n=28)$ and apoprotein distribution after separation of plasma by gelfiltration $(n=3)$

\begin{tabular}{|c|c|c|c|c|}
\hline & VLDL & $\mathrm{IDL}+\mathrm{LDL}$ & $\mathrm{HDL}$ & Plasma \\
\hline \multicolumn{5}{|l|}{ apo C-III } \\
\hline Day 0 & $0.07 \pm 0.02$ & $0.61 \pm 0.05$ & $5.70 \pm 0.15$ & $6.2 \pm 0.3$ \\
\hline Day 7 & $0.66 \pm 0.06$ & $1.58 \pm 0.21$ & $9.00 \pm 0.46$ & $12.7 \pm 1.0$ \\
\hline Day 30 & $0.93 \pm 0.10$ & $1.12 \pm 0.19$ & $9.53 \pm 0.35$ & $10.7 \pm 0.7$ \\
\hline Adults & $1.55 \pm 0.48$ & $1.49 \pm 0.31$ & $10.07 \pm 1.07$ & $11.9 \pm 0.6$ \\
\hline \multicolumn{5}{|l|}{ apo $\mathrm{E}$} \\
\hline Day 0 & $0.04 \pm 0.01$ & $0.64 \pm 0.11$ & $2.92 \pm 0.35$ & $3.5 \pm 0.3$ \\
\hline Day 7 & $0.66 \pm 0.20$ & $1.12 \pm 0.18$ & $3.36 \pm 0.23$ & $5.3 \pm 0.2$ \\
\hline Day 30 & $0.53 \pm 0.14$ & $1.42 \pm 0.17$ & $2.41 \pm 0.29$ & $4.1 \pm 0.1$ \\
\hline Adults & $0.57 \pm 0.03$ & $0.94 \pm 0.16$ & $2.92 \pm 0.69$ & $4.1 \pm 0.2$ \\
\hline
\end{tabular}




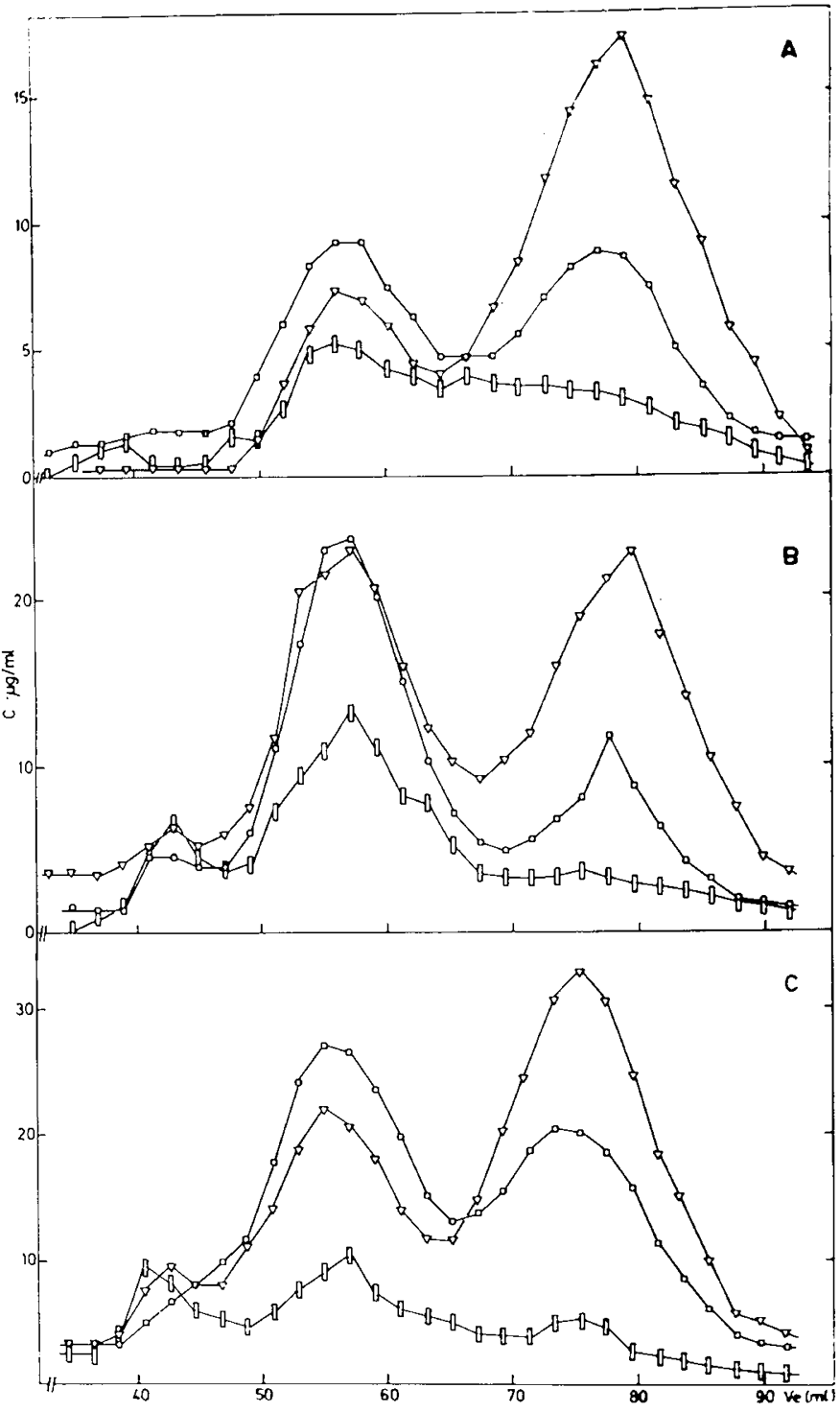

Fig. 2. Lipid profiles of newborn lipoproteins separated by gel filtration, at A: 0, B: 7 , and C: 30 days. $\square$, cholesterol; $\square$, triglycerides; $\nabla$, phospholipids.

AI/AII molar ratio in $\mathrm{HDL}$ as a function of the particle size, is characterized by two maxima corresponding to $\mathrm{HDL}_{2}$ and $\mathrm{HDL}_{3}$ with values around 3.5-4.

Plasma fractionation by ultacentrifugation in a density gradient enabled the resolution of HDL into three subclasses at densities between 1.063 and $1.10 \mathrm{~g} / \mathrm{ml}\left(\mathrm{HDL}_{2 \mathrm{~b}}\right), 1.10-1.15 \mathrm{~g} / \mathrm{ml}$ $\left(\mathrm{HDL}_{2 \mathrm{a}+3 \mathrm{a}}\right)$, and $1.15-1.21 \mathrm{~g} / \mathrm{ml}\left(\mathrm{HDL}_{3 \mathrm{~b}+\mathrm{c}}\right)(8)$. An $\mathrm{HDL}_{1}$ fraction (11), containing small amounts of apo AI and apo AII, was also identified at lower densities $(1.050-1.063 \mathrm{~g} / \mathrm{ml})$. We observed a distinct evolution of these HDL subclasses in newborns. In the $\mathrm{HDL}_{1}$ and $\mathrm{HDL}_{2 \mathrm{~b}}$ subfractions, apo AI and apo AII decreased slightly between 0 and 7 days. The apo AI concentration subsequently increased between 7 and 30 days while the apo AII levels remained constant. In the $\mathrm{HDL}_{2 \mathrm{a}+3 \mathrm{a}}$ and $\mathrm{HDL}_{3 \mathrm{~b}+c}$ fractions, the concentrations of all components increased with age and reached adult values at 30 days. In agreement with the gel filtration data these increases were most pronounced between 7 and 30 days.

The mean apo AI/AII molar ratio throughout the HDL density range was calculated in six newborns at 0,7 , and 30 days and compared to that in adults. The apo AI/AII ratio is maximal at lower densities (HDL ${ }_{2 b}$ subfraction) and varies with the age of the newborn. It decreases between 0 and 7 days and subsequently increases between 7 and 30 days. Adult values are intermediary between 0 and 30 day levels. The apo AI/AII ratio observed in the $\mathrm{HDL}_{1}$ and $\mathrm{HDL}_{2 \mathrm{~b}}$ subclasses, isolated by ultracentrifugation, is significantly higher than in the corresponding fractions separated by gel filtration. The apo Al concentrations were comparable in both techniques, whereas the apo AII concentrations were significantly lower after ultracentrifugation, especially in the $\mathrm{HDL}_{1}$ fraction.

\section{DISCUSSION}

Herein we further documented the apoprotein and lipid composition of lipoprotein subfractions in newborns at 0,7 , and 30 days as longitudinal data are required for a meaningful interpretation of lipoprotein profiles in newborns. At birth, VLDL and LDL concentrations are low, while HDL represents the major lipoprotein fraction. All lipoproteins are enriched with phospholipids, possibly due to high amounts of phospholipid-rich, nascent-like HDL, from which phospholipid transfer to other lipoproteins might occur.

The evolution of the plasma apoprotein concentrations between 0 and 30 days suggests that two groups of apoproteins behave differently. Apo B and apo CIII levels are low at birth, increase about 2 -fold between 0 and 7 days, and then remain

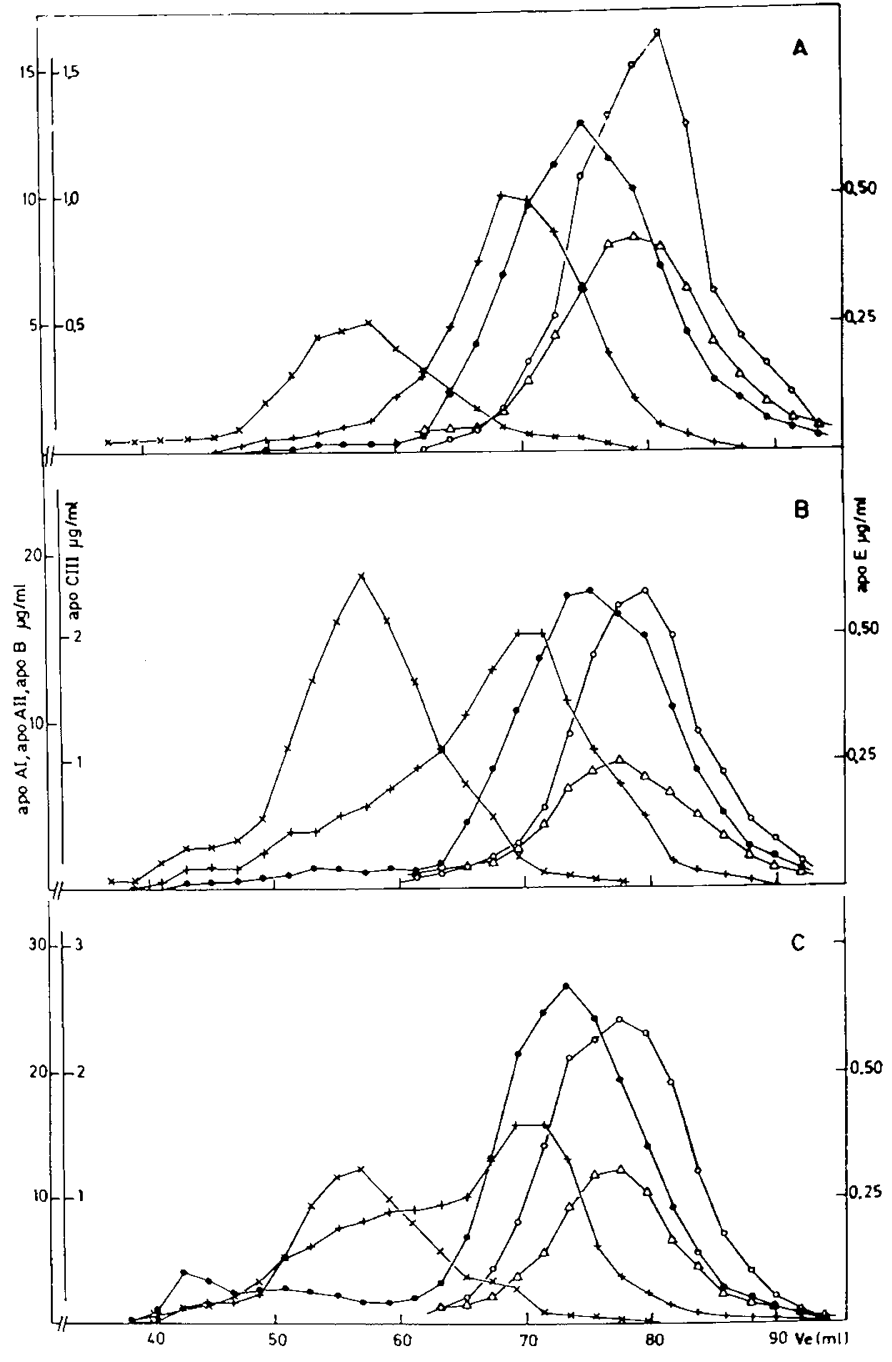

Fig. 3. Apoprotein profiles of newborn lipoproteins separated by gel filtration, at A: 0, B: 7, C: 30 days. $O$, apo AI; $\triangle$, apo AII; X, apo B; apo $\mathrm{CIII} ;+$, apo $\mathrm{E}$. 


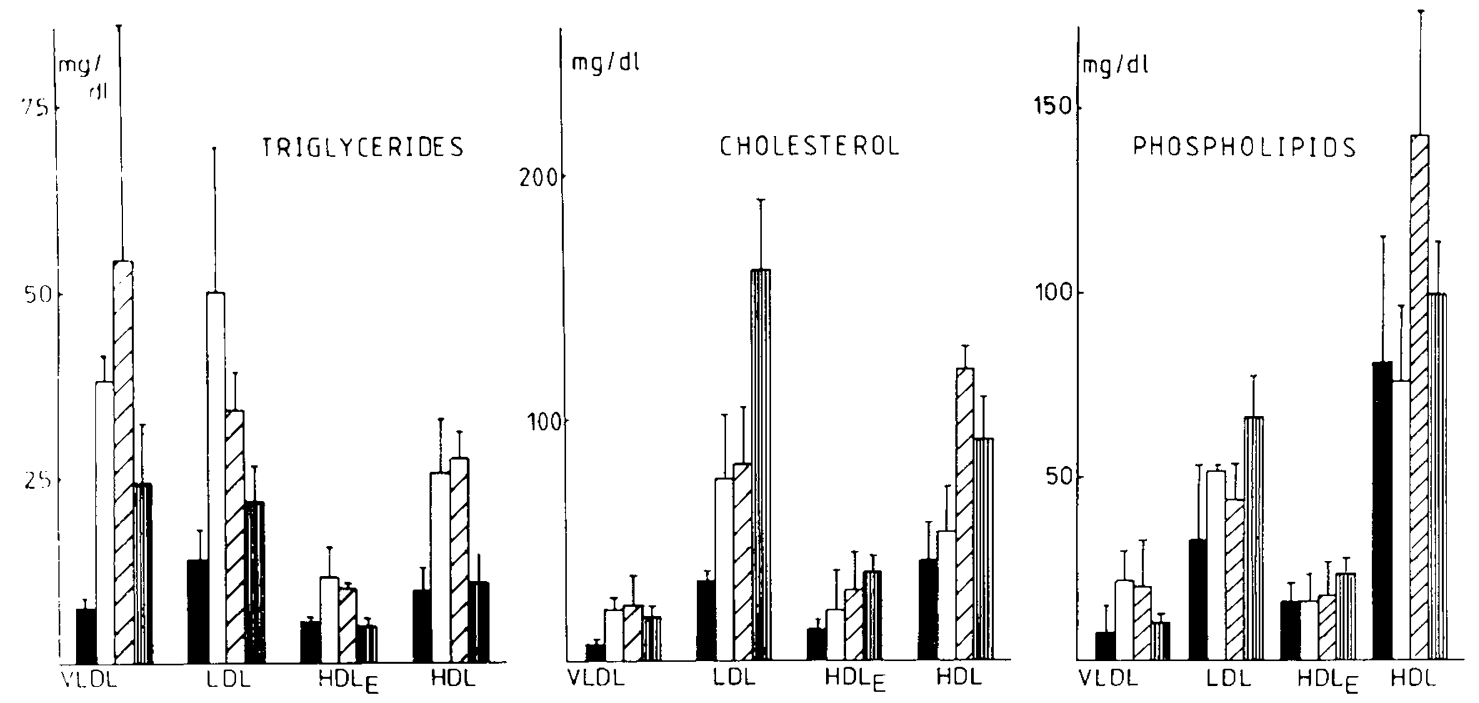

Fig. 4. Concentrations of lipids in lipoproteins separated by gel filtration. $\mathrm{HDL}_{\mathrm{E}}$ corresponds to the apo E-rich HDL. Symbols as in Figure 1.
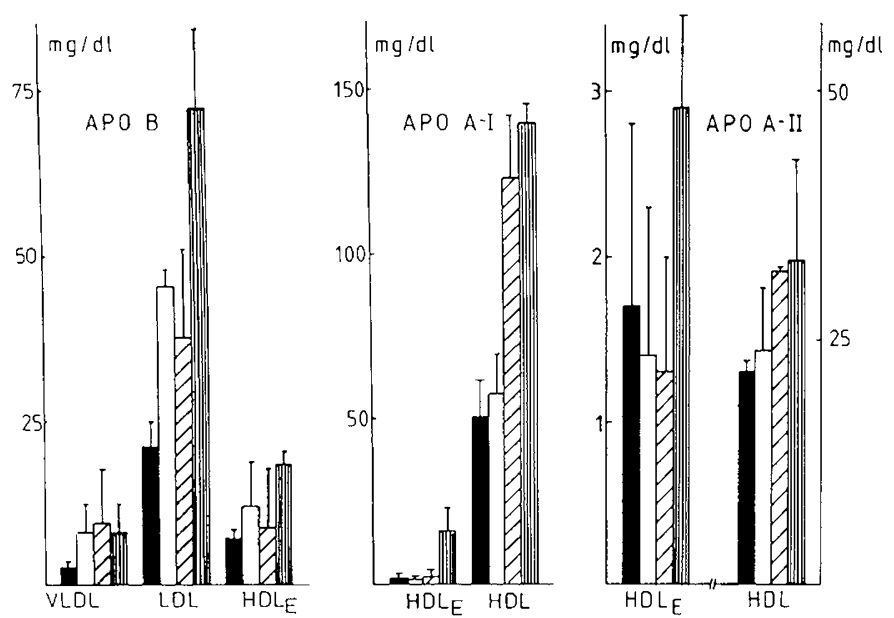

Fig. 5. Concentrations of apolipoproteins AI, AII, and B in lipoproteins separated by gel filtration. Symbols as in Figure 1.

stabile. Apo AI and apo AII, which are present at high concentrations at birth ( 60 and $80 \%$ of the adult values respectively), increase most significantly between 7 and 30 days.

The apo E concentrations measured in cord-blood differ widely among literature reports $(3,4,7,12-14)$. Normal to subnormal apo E levels were estimated by radioimmunoassay (7) and EIA $(3,4,12)$ whereas elevated apo E concentrations were measured by others using radioimmunoassay (13) and competitive ELISA (14). Our values did not differ significantly from those of an adult control group. Plasma apo $E$ increases at 7 days due to increased VLDL synthesis and slightly decreases at 30 days mostly in HDL.

In neonates, an apo E-rich HDL fraction (19) containing relatively more apo $\mathrm{E}$, AII, and CIII and less apo AI could be detected. The HDLE concentration decreases with age; this decrease coincides with the apo $\mathrm{E}$ increase in VLDL, suggesting a transfer of apo E from HDL to VLDL since the plasma apo E level remains constant. These observations support the hypothesis of Innerarity et al. (7), according to whom apo E-rich HDL interact with the LDL (apo B, E)-receptor at birth to compensate for the low LDL concentrations. As VLDL synthesis and catabolism become active between 0 and 7 days, HDL $\mathrm{E}_{\mathrm{E}}$ progressively loses its importance for cholesterol delivery to peripheral cells.

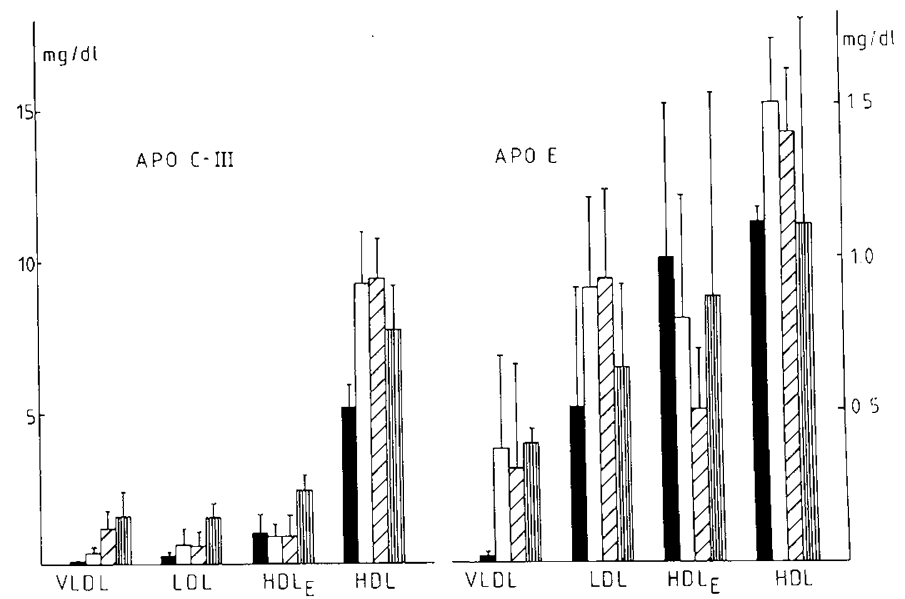

Fig. 6. Concentration of apoliproteins CIII and E in lipoproteins separated by gel filtration. Symbols as in Figure 1.

The HDL fraction, isolated in a density range $1.050-1.063 \mathrm{~g} /$ $\mathrm{ml}$, contained less apo AII and apo E than the corresponding $\mathrm{HDL}_{\mathrm{E}}$ fraction isolated by gel filtration. This suggests that either apo AII is released together with apo E, possibly as an apo E-AII complex (15), or that different kinds of particles are isolated by the two techniques. The distribution of the HDL subclasses shows that while $\mathrm{HDL}_{2 \mathrm{~b}}$ is relatively high at birth, the major apo $\mathrm{AI}$ and apo AII increase between 7 and 30 days, occurs in the HDL $_{3 b+3 c}$ subfraction, which contains mostly nascent HDL particles. Despite a high hepatic lipase activity (17), the $\mathrm{HDL}_{2 b}$ concentration measured at birth is high compared to that at 7 and 30 days. Differences in the phospholipid content and in the apo AI/AII ratio of the $\mathrm{HDL}_{2 b}$ particles might influence their substrate properties and account for these differences.

In summary we can state that, in newborns plasma lipoprotein patterns undergo significant changes in terms of their distribution and composition. The regulatory function of several apoproteins and their interaction with receptors are likely to be different early in life compared to the adult situation. After 1 month of life, although the absolute plasma concentrations of apo CIII, E, and $\mathrm{B}$ are close to those measured in adults, the distribution of the apoproteins between VLDL, LDL, and HDL is still significantly different. Whether these differences reflect the adaptability of the infants to specific dietary regimes, consisting mostly of fat, or 
might represent steps in the slow evolution from the patterns in newborns toward adult profiles is not clear. Detailed studies of lipoprotein patterns in infants fed different diets should provide some answers to this question.

\section{REFERENCES}

1. Van Biervliet JP, Vercaemst R, De Keersgieter W, Vinaimont $N$, Caster $H$, Rosseneu M 1980 Evolution of lipoprotein patterns in newborns. Acta Pediatr Scand 69:593-596

2. Van Biervliet JP, Vinaimont N, Caster H, Vercaemst R, Rosseneu M 1981 Plasma apoprotein and lipid patterns in newborns. Influence of nutritional factors. Acta Pediatr Scand 70:851-856

3. McConathy WJ, Lane DM 1980 Studies on the apolipoproteins and lipoproteins of cord serum. Pediatr Res 14:757-761

4. Lane DM, McConathy WJ 1983 Factors affecting the lipid and apolipoprotein levels of cord sera. Pediatr Res 17:83-91

5. Davis PA, Forte TM 1981 Neonatal umbilical cord blood lipoproteins. Isolated and characterization of intermediate density and low density lipoproteins. Arteriosclerosis 2:37-43

6. Van Bicrvliet JP, Rossencu M, Caster H, Vinaimont N Dependence of plasma lipoprotein distribution and composition in newborns on the type of diet. Eur J Pediatr (in press)

7. Innerarity TL, Bersot TP, Arnold KS, Weisgraber KH, Davis PA, Forte TM, Mahlcy RW 1984 Receptor binding activity of high density lipoproteins containing apoprotein $\mathrm{E}$ from abetalipoproteinemic and normal neonate plasma. Metabolism 33:186-195

8. Rosseneu M, Van Biervliet JP, Bury J, Vinaimont N 1983 Isolation and characterisation of lipoprotein profiles in newborns by density gradient ultracentrifugation. Pediatr Res 17:788-794

9. Bury J, Rosseneu M 1985 Enzyme linked immunosorbent assay for human apolipoprotein CIII. J Clin Chem Clin Biochem 23:63-68

10. Bury J, Vercaemst R, Rosseneu M, Belpaire F Quantitation of human apolipoprotein $E$ by enzyme-linked imunosorbent assay. Clin Chem (in press)

11. Schmitz G, Assman G, Melnik B 1981 The role of lecithin:cholesterol acyl transferase in $\mathrm{HDL}_{3} / \mathrm{HDL}_{2}$ interconversion. Clin Chim Acta 119:225-236

12. Dolphin PJ, Beckenbridge WC, Dolphin MA, Tan MH 1984 The lipoproteins of human umbilical cord blood; apolipoprotein and lipid levels. Atherosclerosis 51:109-122

13. Blum CB, Davis PA, Forte TM 1983 Elevated levels of apolipoprotein E in the high density lipoproteins of human cord blood plasma. J Lipid Res 26:755760

14. Meunier S, Gambert P, Lallemant C, Milne RW, Marcel YL 1984 Dosage immunologique de l'apoproteine $\mathrm{E}$ dans le serum humain "Les Journees du GERLI." Touquet 6:81(abstr)

15. Weisgraber K, Mahley RW 1978 Apoprotein (E-AII) complex of human plasma lipoproteins. I. Characterization of this mixed disulfide and its identification in a high density lipoprotein subfraction. J Biol Chem 253:6281-6288

16. Rovamo L, Nikkila EA. Taskinen MR, Raivio KO 1984 Postheparin plasma lipoprotein and hepatic lipases in preterm neonates. Pediatric Res 18:11041107 